\title{
Vasopressors and inotropes in cardiogenic shock: is there room for "adrenaline resuscitation"?
}

\author{
Nuccia Morici ${ }^{1 *}$, Miriam Stucchi ${ }^{1}$, Alice Sacco ${ }^{1}$, Maurizio A. Bottiroli ${ }^{2}$, Fabrizio Oliva ${ }^{1}$ and on behalf of the AltShock \\ group
}

See related research by Tarvasmäki et al. http://ccforum.biomedcentral.com/articles/10.1186/s13054-016-1387-1

We read with interest the paper of Tarvasmäki et al. [1] regarding the role of inotropes and vasopressors in patients with cardiogenic shock. The authors should be congratulated for their effort of prospectively collecting a huge amount of data in one of the most challenging settings. However, too much emphasis seems to be placed on the authors' conclusions, especially taking into account some of the study's limitations.

Right from the start of the paper, a fearsome association between adrenaline and short-term mortality in the generic setting of cardiogenic shock is described. However, up to $80 \%$ of the patients enrolled in the study had an acute coronary syndrome as the cause leading to shock and this should have been emphasized [2].

Adrenaline doses (maximum infusion rate $0.22 \mathrm{mcg} /$ $\mathrm{kg} / \mathrm{min}$ (interquartile range $0.10-0.36$ )) were abnormally high. It is well known that, depending on vascular beds and concentration, adrenaline may induce either vascular dilatation or contraction, with the vasopressor effects arising at higher doses [3]. These effects might be extremely amplified when coupled with other vasopressors, leading to impaired organ functions. In this study, patients receiving adrenaline were also more likely to receive higher doses of noradrenaline and dopamine.

Furthermore, patients receiving adrenaline were more frequently resuscitated prior to admission, had a higher incidence of low output state, and worse renal function at admission. A more frequent recourse to a mechanical assist device was also present in these patients. Considering the small sample size (40 patients received adrenaline), might propensity score and multivariable analysis take account of all these confounding and effect modifiers? It is well known that researchers should use extreme caution when interpreting the results of analyses performed including a propensity score as a covariate in a multivariable model [4].

Looking at the unadjusted odds ratios reported in their Fig. 1 [1], we were impressed by the confidence intervals. Levosimendan was used in 52 patients: this sample is small but the effect estimation is accurate because these patients were, presumably, clinically selected. Noradrenaline was used in 162 patients: this sample is larger but the effect estimation is less accurate because, supposedly, these patients were a more heterogeneous group. What about the patients receiving adrenaline? Were they at the extreme spectrum of the population enrolled?

We believe there is not enough evidence to promote a link between adrenaline administration and increased death rates. Conversely, low to mid doses of vasopressors might be considered part of an integrated approach but only when massive myocardial damage has not yet occurred.

* Correspondence: nuccia.morici@ospedaleniguarda.it

'De Gasperis Cardio Center, Niguarda Ca' Granda Hospital, Milan, Italy

Full list of author information is available at the end of the article 


\title{
Authors' response to "Vasopressors and inotropes in cardiogenic shock: is there room for 'adrenaline resuscitation'?"
}

\author{
Tuukka Tarvasmäki, Johan Lassus, Alexandre Mebazaa and Veli-Pekka Harjola
}

We thank Morici and colleagues for their interest in our paper and acknowledging our work. They point out that most patients in our study had cardiogenic shock (CS) caused by acute coronary syndrome. This is clearly stated in the results and also described in the previously published paper on the characteristics and outcome of this prospectively enrolled cohort of CS [5].

The dose of adrenaline and concomitant use of other vasopressors and inotropes may indeed be subject to variation according to local experience and practices. In a contemporary randomized controlled trial in CS, IABP-SHOCK II, the median dose of adrenaline was $0.3 \mu \mathrm{g} / \mathrm{kg} / \mathrm{min}$ (Table S1 in the Supplementary Appendix of [6]), which is very similar to the dose recorded in the CardShock study. However, the association of adrenaline with mortality was not dependent on the doses of other vasopressors. The characteristics of patients treated with and without adrenaline are also shown in Table 1 in our paper [1] and mortality analyses were adjusted for differences. Mechanical assist devices other than an intra-aortic balloon pump were used in very few patients overall.

We are aware of the limitations regarding propensity score adjustment. Therefore, we performed additional propensity score matching as a sensitivity analysis, which confirmed the finding. In addition, further adjustment with the use of intra-aortic balloon pump or other mechanical assist devices did not change the results. While the study population is of limited size and the estimates of treatment effects may be susceptible to bias by unknown or unmeasured variables, we think that the results are consistent. All in all, while our study does not prove causality, it does raise safety concerns about using adrenaline in CS.

\section{Abbreviation \\ CS: Cardiogenic shock}

\section{Acknowledgements}

The AltShock group: Stefano Carugo, Diego Castini, Emanuele Catena, Manlio Cipriani, Elena Corrada, Maria Frigerio, Maria Pia Gagliardone, Andrea Garascia, Francesco Gentile, Antonio Mafrici, Filippo Milazzo, Marco Negrini, Federico

Pappalardo, Claudio Russo, Michele Senni, Romano Giuseppe Seregni.

\section{Authors' contributions}

$\mathrm{NM}, \mathrm{MS}, \mathrm{AS}, \mathrm{MB}$, and FO designed the paper, participated in drafting the manuscript, and have read and approved the final version.

\section{Competing interests}

The authors declare that they have no competing interests.

\section{Author details}

'De Gasperis Cardio Center, Niguarda Ca' Granda Hospital, Milan, Italy.

${ }^{2}$ Anestesia e Rianimazione 3, Niguarda Ca' Granda Hospital, Milan, Italy.
Published online: 27 September 2016

References

1. Tarvasmäki T, Lassus J, Varpula M, Sionis A, Sund R, Køber L, Spinar J, Parissis J, Banaszewski M, Silva Cardoso J, Carubelli V, Di Somma S, Mebazaa A, Harjola VP, CardShock study investigators. Current real-life use of vasopressors and inotropes in cardiogenic shock - adrenaline use is associated with excess organ injury and mortality. Crit Care. 2016;20:208-19.

2. Nieminen MS, Buerke M, Cohen-Solál A, Costa S, Edes I, Erlikh A, Franco F, Gibson C, Gorjup V, Guarracino F, Gustafsson F, Harjola VP, Husebye T, Karason K, Katsytadze I, Kaul S, Kivikko M, Marenzi G, Masip J, Matskeplishvili S, Mebazaa A, Møller JE, Nessler J, Nessler B, Ntalianis A, Oliva F, Pichler-Cetin E, Pöder P, Recio-Mayoral A, Rex S, Rokyta R, Strasser RH, Zima E, Pollesello P. The role of levosimendan in acute heart failure complicating acute coronary syndrome: a review and expert consensus opinion. Int J Cardiol. 2016;218:150-7.

3. Shen B, Leung YK, Kwok YC, Kwan HY, Wong CO, Chen ZY, Huang Y, Yao X. Epinephrine-induced $\mathrm{Ca} 2+$ influx in vascular endothelial cells is mediated by CNGA2 channels. J Mol Cell Cardiol. 2008;45:437-45.

4. Hade EM, Lu B. Bias associated with using the estimated propensity score as a regression covariate. Stat Med. 2014;33:74-87.

5. Harjola VP, Lassus J, Sionis A, Kober L, Tarvasmaki T, Spinar J, et al. Clinical picture and risk prediction of short-term mortality in cardiogenic shock. Eur J Heart Fail. 2015;17:501-9. doi:10.1002/ejhf.260.

6. Thiele H, Zeymer U, Neumann FJ, Ferenc M, Olbrich HG, Hausleiter J, et al. Intraaortic balloon support for myocardial infarction with cardiogenic shock. N Engl J Med. 2012;367:1287-96. doi:10.1056/NEJMoa1208410. 